\title{
Rapidly evolving SNPs feature highly significant trait associations in GWAS SNP hotspots
}

\author{
Roman Babenko \\ Laboratory of Neuropathology Modeling, \\ Institute of Cytology and Genetics SB RAS \\ Novosibirsk, Russia \\ babe-roman@yandex.ru
}

\author{
Anton Zhuravlev \\ Laboratory of Eurasian paleogenetics, \\ Institute of Cytology and Genetics SB RAS \\ Novosibirsk, Russia \\ zhuravlev@bionet.nsc.ru
}

\begin{abstract}
As previously observed, highly significant GWAS related SNPs are regularly clustered with other associated SNPs, implying haplotype mediated mode of association. We compiled a set of enriched GWAS SNPs 'hotspot' regions and worked out a strategy to elucidate population specific haplotype spectra. We present several regions in demonstrating specific effects of these haplotypes. In particular, there is an elevated genetic drift rate in allele frequencies. Asian populations demonstrate the highest homogeneity in haplotype variation, while European populations often fix a unique allele not present in other populations, and usually maintaining deleterious effect, at least considering BMI trait. Also, the regions are non-randomly resided in open chromatin nuclear compartments, and manifest $\mathrm{Hi}-\mathrm{C}$ interactions in a range of cases.
\end{abstract}

Keywords - GWAS, SNP, association, obesity, selective sweep, continental populations, $1000 \mathrm{G}$

\section{Introduction}

The need for GWAS SNPs annotation remains an acute task in the course of elucidating the casual variants invoking disease pathology and trait manifestation. Many tools have been created for that task: GwasRap, FUMA, SNAP (Nishizaki, Boyle, 2017 [1]). Once the majority of GWAS SNPs reside in non-coding regions, the major approach for elucidating SNPs functional impact on trait currently results in employing many data repositories, such as DNA variation sources based on 1000 Genomes Project, International HapMap Project, annotations of functional elements [2], and conservation information derived from multiple species alignments $[2,3]$. Chromatin state markers (DNAse accessibility profiles, histone marks landscape, Hi-C maps, etc) are employed by such programs as FunciSNP, Haploreg, GWAS3D [1].

One of the crucial factor of disease related locus is its rapid evolution rate including selection sweeps [4]. It can be assessed by tracing the locus structure dynamics in the course of evolution of modern continental supergroups (EAS, SAS, EUR, AFR). For example, Hapmap project delineates the haploblock structure for each particular supergroup [3]. But the methods for qualitative/quantitative comparison of these cannot be immediately found. We implemented a pipeline to elucidate the most drastic differences between supergroups haploblock structures based on automated selection of the most discriminative GWAS SNPS (evoTAG SNPs) for these. Consequently, based on haploblock dynamics we were able to reconstruct evolutionary events underlining the current alleles structure in the locus.
Research

We constructed the pipeline for extracting the maximal population specific variance SNPs within GWAS hotspots. Based on the methodology we present the analysis of 7 GWAS SNPs hotspot loci with quite rapid fixation of the disease associated SNPs. The proposed method may help in elucidating the casual SNPs, as well as it underscores high disease risk populations across supergroups.

\section{Conclusion}

Intriguingly, but expectedly, when we applied the pipeline to the region of chromosome 1 we found that the most informative SNPs from 455 total in the region are 6 GWAS annotated. It underscores the adequacy and utility of the method

\section{ACKNOWLEDGMENT}

The work was supported by Russian Foundation for Basic Research grant 19-2015-2099

\section{REFERENCES}

[1] Nishizaki SS, Boyle AP. Mining the unknown: Assigning Function to Noncoding Single Nucleotide Polymorphisms. Trends Genet. 2017 Jan;33(1):34-45. doi:10.1016/j.tig.2016.10.008.

[2] Luo Y, Hitz BC, Gabdank I, Hilton JA, Kagda MS, Lam B, Myers Z, Sud P, Jou J, Lin K, Baymuradov UK, Graham K, Litton C, Miyasato SR, Strattan JS, Jolanki O, Lee JW, Tanaka FY, Adenekan P, O'Neill E, Cherry JM. New developments on the Encyclopedia of DNA Elements (ENCODE) data portal. Nucleic Acids Res. 2020;48(D1):D882-D889. doi: 10.1093/nar/gkz1062

[3] 1000 Genomes Project Consortium, Abecasis GR, Altshuler D, Auton A, Brooks LD, Durbin RM, Gibbs RA, Hurles ME, McVean GA. A map of human genome variation from population-scale sequencing. Nature. 2010 Oct 28;467(7319):1061-73. doi: 10.1038/nature09534.

[4] Refoyo-Martínez A, da Fonseca RR, Halldórsdóttir K, Árnason E, Mailund T, Racimo F. Identifying loci under positive selection in complex population histories. Genome Res. 2019:1506-1520. doi: 10.1101/gr.246777.118. 